\title{
Preparación y caracterización de nanofluidos: Influencia de variables sobre su estabilidad, estado de aglomeración y propiedades físicas
}

\author{
ROSA MONDRAGÓN ${ }^{1 *}$, J. ENRIQUE JULIÁ${ }^{1}$, ANTONIO BARBA ${ }^{2}$, JUAN CARLOS JARQUE ${ }^{2}$ \\ ${ }^{1}$ Departamento de Ingeniería Mecánica y Construcción. \\ ${ }^{2}$ Departamento de Ingeniería Química e Instituto Universitario de Tecnología Cerámica. \\ Universitat Jaume I. Campus de Riu Sec. 12071-Castellón de la Plana. España \\ *correspondencia/corresponding author: e-mail: rosa.mondragon@ uji.es
}

\begin{abstract}
En los últimos años se ha extendido el uso de suspensiones conteniendo partículas de tamaño nanométrico, conocidas como nanofluidos, en múltiples aplicaciones debido las buenas propiedades que presentan los materiales nanoparticulados. Una de las principales ventajas que presenta el uso de nanofluidos es su elevada estabilidad, que hace que las partículas no sedimenten durante largos periodos de tiempo. Esta estabilidad depende de las condiciones de preparación tales como el pH del medio, la presencia de electrolitos en el medio o el contenido en sólidos. Por otra parte, existen una serie de propiedades físicas que se ven influenciadas y alteradas por el estado de aglomeración de las partículas. En este artículo se analizarán todas las variables que influyen en el estado de aglomeración de las partículas y la estabilidad de los nanofluidos, así como las propiedades a partir de las cuales se puede obtener información sobre el estado de la suspensión. Además, se exponen los diferentes métodos de dispersión de nanopartículas y su efectividad.
\end{abstract}

Palabras clave: Suspensiones, Nanopartículas, Reología, Caracterización.

Preparation and characterization of nanofluids: Influence of variables on its stability, agglomeration state and physical properties

In recent years it has spread the use of suspensions containing nanometre sized particles, known as nanofluids, in many applications owing the good properties having nanocrystalline materials. One of the main advantages of using nanofluids is its high stability, which causes the particles do not settle over long periods of time. This stability depends on the preparation conditions such as $\mathrm{pH}$, the presence of electrolytes or the solids content. Moreover, there are a number of physical properties which are influenced and altered by agglomeration of the particles. This article will analyze all the variables that affect agglomeration of the particles, nanofluids stability and properties from which it can obtain information on the state of suspension. It then lays out the different methods of dispersion of nanoparticles and their effectiveness.

Keywords: Suspensions, Nanoparticles, Rheology, Characterization.

\section{INTRODUCCIÓN}

Los avances tecnológico desarrollados en los últimos años han permitido la obtención y el uso de nuevos materiales de tamaño nanométrico. En la mayoría de los casos, estos materiales sólidos son puestos en suspensión en un medio acuoso u orgánico y utilizados en múltiples aplicaciones: sistemas de transferencia de calor, captadores solares, almacenamiento de energía, secado por atomización para obtención de gránulos nanoestructurados, etc.

Se entiende por ciencia coloidal el estudio de los sistemas que contienen unidades cinéticas que son grandes en comparación con las dimensiones atómicas (1). Estos sistemas pueden ser aquellos en los que las partículas se mueven libremente en todas las direcciones, o pueden ser sistemas derivados, como coágulos o geles, en los que las partículas han perdido su movilidad entera o parcialmente, pero mantienen su individualidad. El estado coloidal de subdivisión comprende partículas con un tamaño suficientemente pequeño para no verse afectadas por las fuerzas gravitacionales $(\leq 1 \mu \mathrm{m})$ pero suficientemente grande para mostrar diferencias con respecto de las disoluciones reales (>1 nm). Las partículas coloidales pueden ser de naturaleza cristalina o amorfa. Dentro del estado coloidal de subdivisión, se denominan nanopartículas aquellos materiales con un tamaño inferior a $100 \mathrm{~nm}$.

Las suspensiones acuosas de partículas de tamaño comprendido entre 5-100 nm son de gran interés en muchas aplicaciones. En 1995, Choi, utilizo por primera vez el término nanofluidos para designar a los fluidos con nanopartículas en suspensión (2). Estas suspensiones presentan importantes 
ventajas con respecto a las suspensiones coloidales convencionales como su elevada superficie específica, el predominio del movimiento Browniano, reducción de la obturación de canales por los que circula y fácil modificación de sus propiedades ajustando las interacciones entre partículas. Pero una de sus principales características es que pueden aguantar durante periodos de tiempo muy prolongados sin que se produzca una sedimentación significativa o una pérdida de estabilidad.

El término estabilidad en ciencia coloidal es usado no sólo en el sentido termodinámico sino también en un sentido estrictamente coloidal. "Coloidalmente estable" implica que las partículas sólidas no sedimentan ni se agregan a una velocidad significativa (1). Las suspensiones pierden su estabilidad por agregación de las partículas coloidales. Éstas pueden unirse o agregarse por gelificación (formación de una red coherente de partículas que ocupan todo el volumen, y por acción capilar retiene el líquido), coagulación (formación de grupos d partículas compactos en los cuales la concentración es mayor que en la suspensión original) o floculación (unión de partículas mediante puentes formados por un agente floculante formando una estructura abierta y voluminosa) (Figura 1).

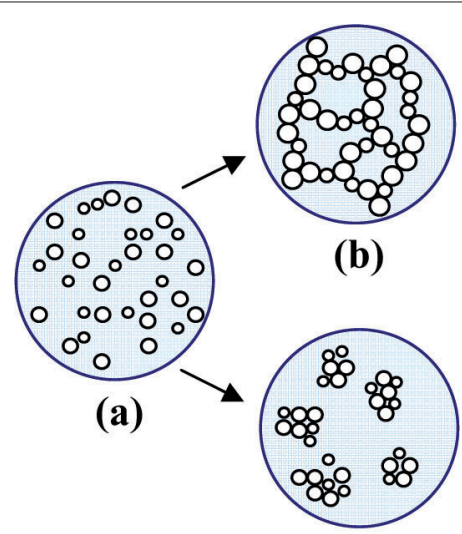

(c)

Figura 1. (a) Sol, (b) gel y (c) floculación y precipitación.

Existe una diferencia básica entre la gelificación y la coagulación o floculación. Ambos implican la unión de partículas y la formación de redes tridimensionales. Pero cuando un sol se gelifica, en primer lugar se vuelve viscoso y posteriormente se vuelve rígido ocupando todo el volumen inicialmente ocupado por el sol. En cambio, cuando un sol se coagula o flocula, se forma un precipitado. Estas diferencias son apreciables principalmente en soles. En mezclas concentradas se puede distinguir un gel, el cual es rígido, pero no entre una suspensión coagulada y una floculada, aunque estos últimos sean resultado de mecanismos de aglomeración diferentes.

Muchos han sido los esfuerzos por determinar la estabilidad de los nanofluidos, el estado de aglomeración de las partículas y los factores que influyen en ellos, para con ello poder optimizar las propiedades físicas que se ven modificadas al variar el estado de aglomeración y así producir suspensiones adecuadas para cada aplicación determinada.

\section{DETERMINACIÓN DE LA ESTABILIDAD COLOIDAL. TEORÍA DLVO}

En 1941, Derjaguin y Landau dieron explicación a muchos de los fenómenos complejos implicados en la estabilidad de los agregados en base a las fuerzas de interacción entre partículas coloidales: fuerzas de atracción de Van der Waals y fuerzas de repulsión electrostática. En 1948 Verwey y Overbeek publicaron una teoría de estabilidad de coloides, cuyas ideas eran virtualmente idénticas a las de los autores anteriores. Como suma de los dos trabajos nace la teoría clásica conocida como teoría DLVO (DerjaguinLandau-Verwey-Overbeek) que analiza la estabilidad de suspensiones coloidales a partir de las interacciones entre las superficies de las partículas en un medio, considerando la energía de interacción de las mismas.

Las fuerzas presentes en una suspensión pueden ser hidrodinámicas, coloidales, gravitacionales, inerciales, electroviscosas o fuerzas provocadas por efectos térmicos. En el rango de tamaños comprendido dentro del estado coloidal de subdivisión el ratio superficie/volumen es extremadamente elevado, y por lo tanto todas las propiedades relacionadas con la superficie se acentúan considerablemente de forma que las interacciones se ven dominadas por fuerzas de corto alcance como las de atracción de Van der Waals y las fuerzas superficiales. La ordenación de las partículas en una dispersión coloidal y su estado de aglomeración vienen determinados por la naturaleza de las fuerzas de interacción $(3,4)$.

Siguiendo la teoría DLVO, el potencial total de interacción es la suma de las fuerzas de atracción y las de repulsión. Las fuerzas de atracción se representan siempre por las fuerzas de Van der Waals de corto alcance. Dichas fuerzas tienen su origen en interacciones dipolo-dipolo inducido que dependen de la distancia y de la naturaleza de los átomos. Esta fuerza es proporcional al valor de la constante de Hamaker $\left(A_{e f f}\right)$, la cual es propia de cada sistema. Esta constante es siempre positiva para dos partículas idénticas y por tanto, la interacción es siempre atractiva.

$$
V_{A}=-\frac{A_{e f f}}{6}\left[\frac{2 r^{2}}{a^{2}-4 r^{2}}+\frac{2 a^{2}}{a^{2}}+\mathrm{h} \frac{a^{2}-4 r^{2}}{a^{2}}\right]
$$

donde $a$ es la distancia de separación entre las partículas y $r$ es el radio de las partículas.

Las fuerzas de Van der Waals empiezan a ser importantes cuando la separación entre las partículas es diez veces inferior a su tamaño. Cuando las partículas se aproximan debido al movimiento browniano y se sitúan a una distancia inferior, éstas se atraen y el sistema se aglomera. Para evitar que lleguen a formarse aglomerados es necesario impedir que las partículas entren en contacto. Esto se consigue provocando la aparición en el sistema de fuerzas repulsivas de mayor magnitud y alcance que las atractivas de forma que mantengan las partículas alejadas unas de otras en la medida de lo posible.

Las fuerzas repulsivas provienen de interacciones electrostáticas, estéricas o por capas de hidratación. En general las interacciones electrostáticas son importantes en sistemas acuosos, mientras que las interacciones estéricas son también efectivas en sistemas no acuosos. 


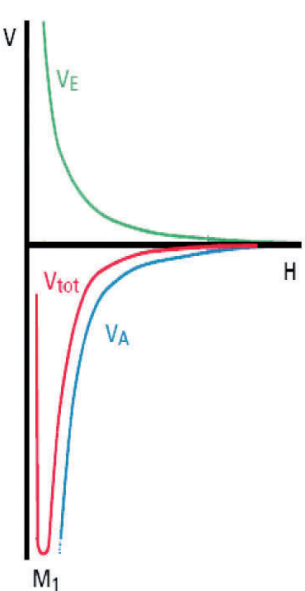

$\mathrm{V}_{\mathrm{A}}$ : Atracción de Van der Waals; $\mathrm{V}_{\mathrm{E}}$ : Repulsión electrostática; $\mathrm{V}_{\text {tot }}=\mathrm{V}_{\mathrm{A}}+\mathrm{V}_{\mathrm{E}}$

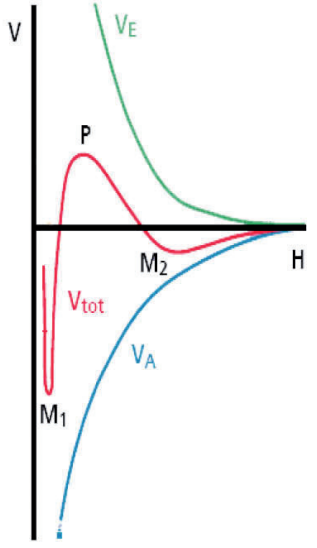

(c)

Figura 2. Potencial total de interacción: (a) Suspensión no estabilizada, (b) Suspensión poco estabilizada, (c) Suspensión bien estabilizada.

- Repulsión electrostática. Las interacciones electrostáticas entre partículas se deben a la aparición de cargas superficiales. La distribución de potenciales determina la energía de interacción entre las partículas y es, en muchos casos, responsable de la estabilidad de la suspensión contra la coagulación. Cuando se aproximan partículas cargadas con cargas del mismo signo se produce una repulsión electrostática entre ellas proporcional al valor de la carga, que las mantiene separadas evitando la formación de agregados de partículas que desestabilizan el sistema.

$$
V_{E}=2 \pi r \varepsilon \varepsilon_{0} \psi_{d} \exp (-\kappa a)
$$

donde $a$ es la distancia de separación entre las partículas, $r$ es el radio de las partículas, $\varepsilon$ es la constante dieléctrica del medio, $\psi_{d}$ es el potencial eléctrico en al superficie de Stern у $\kappa$ es la inversa del espesor de la doble capa eléctrica.

- Repulsión estérica. Es el resultado de la adsorción de cadenas poliméricas y/o surfactantes que se extienden hacia el interior del medio suspensionante impidiendo la aproximación de unas partículas a otras. La repulsión se manifiesta en el momento en que las capas de polímero entran en contacto y empiezas a comprimirse. Depende del número de sitios activos sobre las partículas y del número y posición de los grupos funcionales sobre el polímero, de las interacciones competitivas entre el solvente y otras cadenas de polímero y de la adsorbabilidad de las moléculas de solvente y otros iones en solución.

$V_{S}=\frac{100 \delta}{\pi s^{3}} k T \exp \left(-\frac{\pi a}{\delta}\right)$

donde $a$ es la distancia de separación entre las partículas, $\delta$ es el espesor de la capa polimérica, $s$ es la distancia entre los puntos de adsorción de las cadenas y $k$ es la constante de Boltzmann.

- Repulsión electrostérica. Este tipo de estabilización resulta del efecto combinado de los dos mecanismos anteriores: repulsión estérica y electrostática. Se consigue mediante la adición de polielectrolitos (polímeros cargados) que se adhieren a la superficie de las partículas. De esta forma las cadenas poliméricas actúan generando un impedimento estérico y al mismo tiempo, las cargas presentes al final de las mismas, y en contacto con el medio que las rodea, generan repulsión electrostática entre las partículas. En muchas ocasiones este mecanismo resulta ser el más eficiente.

- Repulsión porhidratación. La estabilización por hidratación es resultado de la fuerte repulsión causada por las fuerzas de hidratación entre partículas en un líquido. Es conocido que alrededor de las superficies hidrófilas en un líquido, existen capas de hidratación con una estructura molecular especial, diferente de la de la masa líquida (5). El espesor de las capas de hidratación depende de la atracción de la superficie por las moléculas del líquido. Cuanto mayor sea la atracción mayor será el espesor de la capa hidratada. Si dos partículas se aproximan suficientemente la una a la otra, las capas de hidratación empiezan a superponerse generando una fuerza repulsiva no electrostática que evita que se aproximen.

La teoría clásica del DLVO propuesta inicialmente solo contempla como fuerzas de interacción las fuerzas de atracción de Van der Waals y las fuerzas de repulsión electrostáticas. El resto de fuerzas de repulsión (estérica, electrostérica y por hidratación) fueron incorporadas con posterioridad en la que se conoce como teoría extendida del DLVO.

La magnitud relativa de todas las interacciones gobierna la estabilidad de las suspensiones. Cuando dos partículas se aproximan, debido a su movimiento aleatorio, en el momento en que se encuentren a una distancia inferior a aquella para la cual la atracción de Van der Waals es efectiva, permanecerán unidas (Figura 2a) a no ser que exista una fuerza de repulsión que lo impida, la cual genera en el potencial total de interacción una barrera energética (Figura 2b,c). Las partículas permanecerán a una distancia de separación para la cual la energía total de interacción es mínima (Punto $M_{1}$ ). Para que la dispersión sea buena, el mínimo debe encontrarse a una separación tal que no exista atracción, y de forma que una 
aproximación de las partículas implique superar la barrera de potencial (Punto $\mathrm{M}_{2}$ ). Si las partículas poseen suficiente energía cinética para superar esta barrera, se producirá la coagulación, mientras que en caso contrario las partículas permanecerán en suspensión. Cuanto mayor sea esta barrera, mayor será la estabilidad de las suspensiones.

\section{FACTORES QUE INFLUYEN EN LA ESTABILIDAD COLOIDAL}

La estabilidad delos nanofluidos y el estado de aglomeración de las partículas puede modificarse mediante el control de las variables de la suspensión que ejercen una influencia sobre las propiedades superficiales de las partículas y, por lo tanto, sobre las fuerzas de interacción entre ellas. Estas variables son el pH de la suspensión, la presencia de electrolitos en el medio y el contenido en sólidos de la suspensión.

\section{1. $\mathrm{pH}$ de la suspensión}

La carga superficial que adquieren las partículas al introducirlas en medio acuoso, depende del $\mathrm{pH}$ de dicho medio (4). Para cada sustancia química existe un $\mathrm{pH}$ al cual el valor de carga superficial de la partícula es cero. Este $\mathrm{pH}$ se conoce como punto de carga cero (pzc).

Sin embargo, cuando las partículas se desplazan, éstas lo hacen junto con la capa de líquido que las rodea, la cual define un plano de cizalla. Todo el material contenido dentro de esta envolvente constituye la unidad cinética de forma que la partícula se mueve junto con parte de líquido que la rodea y la carga que contiene. El análisis de las fuerzas que actúan sobre el sólido o el líquido puede llevarse a cabo en términos de carga o de potencial electrostático. Si se opta por el segundo caso, se obtiene el potencial medio en el plano de cizalla. Este potencial se conoce como potencial zeta. Se denomina punto isoeléctrico (IEP) al $\mathrm{pH}$ para el cual la carga y el potencial en el plano de cizalla (potencial zeta) vale cero.

La disminución o aumento del $\mathrm{pH}$ con respecto al punto isoeléctrico, provoca la adsorción de hidrogeniones o de hidroxilos, adquiriendo las superficies carga positiva o negativa respectivamente. Superficies altamente cargadas, provocan un dominio de las fuerzas de repulsión electrostática sobre las de atracción, aumentando la barrera energética que impide que las partículas se aglomeren. Por lo tanto, cuando mayor sea la diferencia entre el $\mathrm{pH}$ del medio y el $\mathrm{pH}$ correspondiente al punto isoeléctrico del sistema, mayor será la estabilidad del mismo.

\subsection{Presencia de electrolitos}

Cuando la superficie de las partículas se encuentra cargada, dicha carga se ve modificada por la presencia de electrolitos y el correspondiente aumento de la fuerza iónica del medio, I:

$$
I=\frac{1}{2} \sum c_{i} z_{i}^{2}
$$
iónica.
Al introducir un electrolito en una suspensión de partículas cargadas, los contraiones se distribuyen alrededor de las mismas formando una doble capa consistente en una capa interna y una capa difusa. En la capa interna (capa de Stern), los contraiones se encuentran adsorbidos sobre la superficie de las partículas como consecuencia de intentar alcanzar el estado de menor energía eléctrica (Figura 3). Sin embargo, el estado de menor energía termodinámica es aquel en que la concentración de iones es la misma en todos los puntos del sistema, de forma que como resultado de los dos efectos opuestos, se forma una capa difusa de iones débilmente enlazados. Cerca de la superficie habrá un exceso de carga, que se va uniformando a medida que se aleja de la superficie.

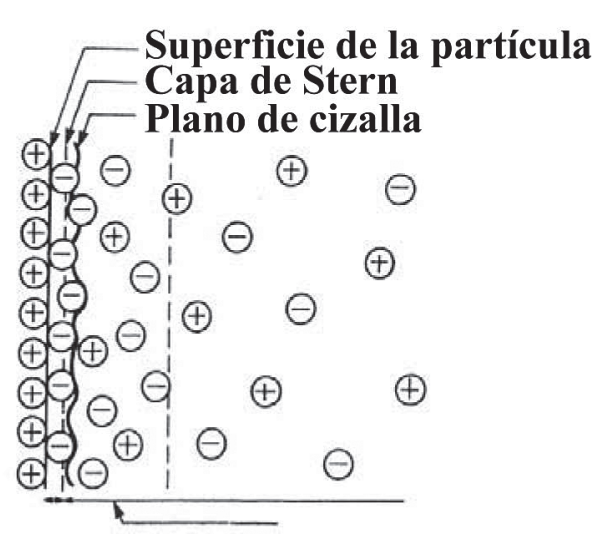

Figura 3. Formación de la doble capa eléctrica.

Cuando las dobles capas de dos partículas se solapan, el potencial eléctrico es más alto de lo normal ya que en esa zona hay una distribución aniónica y catiónica superior a la de equilibrio. El sistema reacciona generándose una presión osmótica que hace que las partículas se repelan.

El espesor de esta doble capa, $\kappa^{-1}$, es inversamente proporcional a la fuerza iónica del medio, de forma que al aumentar la concentración de electrolito la capa se comprime:

$$
\kappa^{-1}=\left[\frac{\varepsilon \varepsilon_{0} R T}{F^{2} \sum c_{i} z_{i}^{2}}\right]^{1 / 2}=\left[\frac{\varepsilon \varepsilon_{0} R T}{F^{2} 2 I}\right]^{1 / 2}
$$

donde $\varepsilon$ es la constante dieléctrica del medio, $R$ es la constante de los gases, $T$ es la temperatura absoluta, $F$ es la constante de Faraday y la fuerza iónica del medio $I$ ha sido definida en la ecuación (4).

Esta disminución del espesor de la doble capa con la fuerza iónica del medio, supone que su efecto se nota a distancias más cortas de forma que la repulsión eléctrica no es capaz de contrarrestar la atracción de Van der Waals formándose aglomerados. Por tanto, un aumento de la concentración de iones en el medio supone la disminución de la barrera de potencial y la formación de aglomerados que desestabilizan la suspensión. Sin embargo, la reducción de espesor de la doble capa provocado por la presencia de electrolitos, hace que disminuya el volumen efectivo de las partículas y que la suspensión se comporte como si estuviera más diluida (6). En este caso, la presencia de un electrolito es positiva siempre 
que no afecte al potencial total de interacción y a la barrera energética.

\subsection{Contenido en sólidos}

La ordenación de las partículas en el sistema depende del espacio ocupado por ellas en relación con el volumen total (3). A bajos contenidos en sólidos, la distancia media entre las partículas es grande en comparación con el radio de las mismas. En este caso, las partículas son capaces de desplazarse libremente a través del medio conducidas por las fuerzas Brownianas. Este régimen se considera como el límite diluido. A medida que la fracción volumétrica aumenta, las interacciones hidrodinámicas así como la probabilidad de colisión entre las partículas se vuelven importantes, favoreciendo los procesos de agregación. A contenidos en sólidos más elevados, las suspensiones coloidales presentan una transición de fase termodinámica. A estos niveles de concentración el movimiento de las partículas individuales se encuentra fuertemente constreñido debido a la presencia de las partículas vecinas.

En suspensiones estabilizadas electrostáticamente, si éstas se encuentran diluidas las fuerzas de repulsión creadas pueden ser suficientes para mantener las partículas alejadas. Sin embargo, cuando la suspensión está concentrada, la falta de espacio hace que esta repulsión pueda no ser suficiente para evitar la aglomeración, formándose un gel muy repulsivo. En esto casos una pequeña adición de electrolitos puede ser favorable ya que disminuye la carga neta de las partículas.

\section{PREPARACIÓN DE NANOFLUIDOS}

En la mayoría de las aplicaciones industriales las nanopartículas se procesan en forma de suspensión y la calidad del producto final depende de las propiedades de estas suspensiones caracterizadas por el tamaño y la morfología de las partículas. Por lo tanto, las nanopartículas que son suministradas normalmente en forma de polvo seco, tienen que ser dispersadas hasta alcanzar una suspensión homogénea y estable. Debido a las fuerzas intensas de Van der Waals, casi todas las nanopartículas se encuentran en forma de aglomerados secos con una dimensión mucho mayor que la de las partículas primarias. Por lo tanto, durante la dispersión es esencial que los grandes agregados, presentes en el polvo seco, se rompan en las nanopartículas primarias o en agregados de tamaño determinado $(7,8)$.

El proceso por el cual las partículas son dispersadas en el líquido juega un papel importante en las propiedades finales. La dispersión y desaglomeración de nanopartículas en líquidos depende de la energía y el tiempo del tratamiento y puede llevarse a cabo en baños de ultrasonidos $(6,9-12)$, sondas de ultrasonidos (13-16), agitadores magnéticos, “agitadores de alta cizalla" (8), molinos de bolas y homogeneizadores. Al comparar varios procedimientos de dispersión de nanopartículas se observa como el tratamiento con sondas de ultrasonidos es el más efectivo de todos (Figura 4) $(15,17,18)$. El tiempo necesario para alcanzar un determinado grado de dispersión depende también del tipo de tratamiento utilizado y de la energía que aplica cada uno de ellos. Así pues, en el caso de los turbodesleidores y los agitadores magnéticos, el tamaño de partícula se modifica de forma importante durante

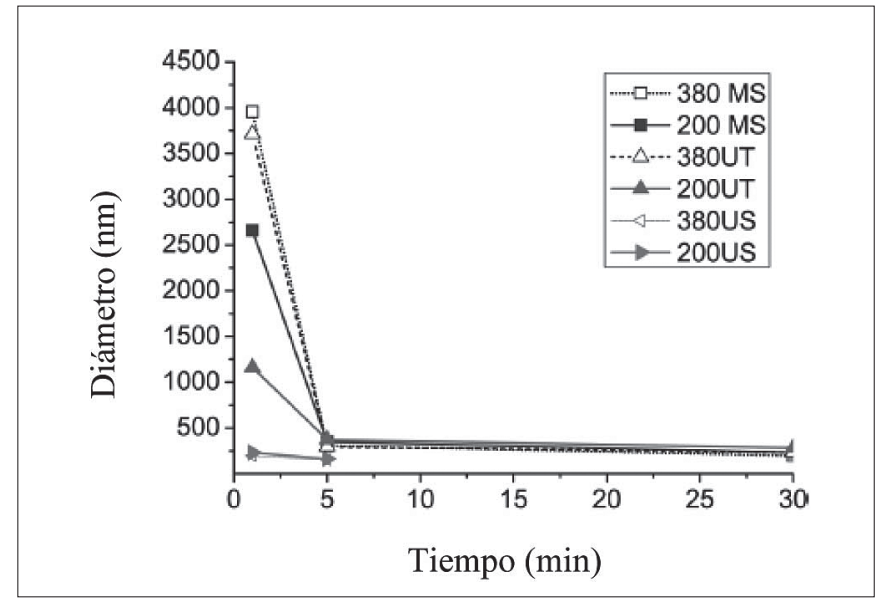

Figura 4. Tamaño medio de partícula obtenido para diferentes tipos de tratamiento en función del tiempo. Símbolos llenos: Aerosil 200, símbolos huecos: Aerosil 380. MS: agitador magnético, UT: turbodesleidor, US: sonda de ultrasonidos (18).

los primeros cinco minutos. A partir de este momento dejan de producirse cambios significativos. En cambio, en el caso de las sondas de ultrasonidos altamente energéticas, el tiempo necesario para que la dispersión sea completa y reproducible es mucho menor, manteniéndose prácticamente invariable el tamaño de agregados a partir del primer minuto de tratamiento.

Sin embargo, una vez cesa la aplicación del tratamiento, las partículas tienden a reaglomerarse debido a las fuerzas de Van der Waals presentes. Éstas hacen que al entrar en contacto las partículas, debido a su movimiento Browniano, se unan formando aglomerados de mayor tamaño. Por lo tanto, una vez concluido el proceso de dispersión es necesario estabilizar las suspensiones si se desea que las partículas se mantengan separadas unas de otras durante un tiempo prolongado. Para ello hay que recurrir a la estabilización electrostática o estérica tal y como predice la teoría DLVO. En general, la estabilización electrostática es preferible en sistemas acuosos y se realiza mediante cambios de $\mathrm{pH}$ conseguidos con la adición de un ácido o una base ( $\mathrm{HCl}$ y $\mathrm{NaOH}$ comúnmente). La estabilización estérica es más utilizada en sistemas no acuosos y se consigue mediante la adición de surfactantes, siendo los más utilizados CTAB, SDS, SDBS, Triton-X100 y sales de amonio o fosfatos.

\section{CARACTERIZACIÓN DE NANOFLUIDOS}

El comportamiento reológico y viscoelástico, así como las propiedades físicas que caracterizan las suspensiones coloidales están directamente relacionados con el estado de agregación de las mismas y dependen, por tanto, de las variables que modifican el potencial total de interacción entre las partículas ( $\mathrm{pH}$ y concentración de electrolito). Es por esto que, a partir de las medidas de todas las propiedades de las suspensiones de nanopartículas, se puede determinar el grado de estabilidad de las mismas y establecer las condiciones que proporcionan suspensiones estables con características adecuadas para su uso o aplicación. Entre las propiedades de interés fácilmente analizables hay que destacar la viscosidad, los módulos elástico y viscoso, el potencial zeta, el tamaño de 
partícula o agregados y la transmisión/ retrodispersión de luz de la muestra.

\subsection{Viscosidad}

El parámetro reológico utilizado por excelencia en el control de los líquidos es la viscosidad, que representa su resistencia al flujo, cuando éste se produce en régimen laminar y en condiciones estacionarias. La viscosidad, $\eta$, se define como la razón entre la tensión de cizalla aplicada a un fluido, $\sigma$, y el gradiente de velocidades que se genera, $\gamma$. Las propiedades reológicas de suspensiones concentradas se ven afectadas principalmente por las fuerzas coloidales entre partículas debido a la fuerte interacción entre ellas. Las fuerzas hidrodinámicas son también importantes, mientras que el movimiento Browniano es más significativo a bajas concentraciones (10).

El comportamiento reológico de suspensiones coloidales está relacionado con el potencial de interacción entre partículas, que a su vez condiciona el grado de estabilidad de las mismas. Así pues, una baja concentración de electrolito o un $\mathrm{pH}$ diferente del punto isoeléctrico generan una elevada barrera energética que se corresponde con una disminución del tamaño de los agregados que fluyen más fácilmente. Por lo tanto, la variación de la viscosidad con el gradiente de velocidad presenta una forma que implica un comportamiento Newtoniano. La suspensión tiene una estructura aleatoria e isotrópica que no cambia significativamente con el gradiente de velocidad. Sin embargo, cuando el sistema se desestabiliza las partículas están tan próximas unas de otras que no se puede despreciar la influencia de las interacciones, dando lugar a la formación de aglomerados de forma que se produce una transición de comportamiento Newtoniano a pseudoplástico. Al mismo tiempo, y siguiendo el mismo criterio de estabilidad, las suspensiones pasan de no presentar un esfuerzo crítico de fluencia a requerir de un esfuerzo inicial para empezar a fluir libremente.

La tensión de fluencia extrapolada es uno de los parámetros más utilizados para estimar el grado de floculación inducido por la presencia de aditivos o por cambios en las condiciones coloidales (19). En suspensiones diluidas, no hay duda de que el fluido fluye fácilmente con la aplicación de bajas cizallas y por lo tanto, no presentan tensión crítica de fluencia. En sistemas más concentrados, en concreto para los materiales como los geles, puede existir una tensión crítica de fluencia ya que se acepta que estos materiales no fluyen libremente por su propio peso (20).

La Figura 5 muestra la interpretación estructural del comportamiento pseudoplástico: éste se debe a la ruptura progresiva de los clusters grandes presentes a bajas cizallas, a medida que ésta se incrementa. La estructura de cualquier suspensión concentrada no puede caracterizarse sólo a partir de su microestructura: los elementos de menor tamaño (como partículas individuales o flóculos primarios) forman grupos más grandes (como clusters, agregados, flóculos, clusters de flóculos...) hasta una estructura expandida (21). El tamaño de estos grupos depende de la cizalla aplicada como consecuencia de las fuerzas hidrodinámicas, conduciendo al concepto de estructura "shear dependent".

En general, para suspensiones estabilizadas o con bajas concentraciones de sólido la reología está gobernada por

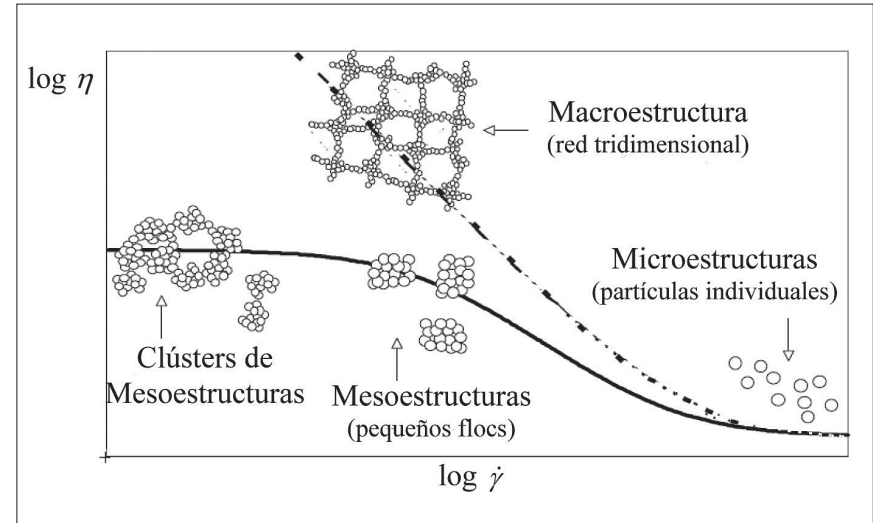

Figura 5. Interpretación estructural del comportamiento pseudoplástico. Ruptura de agregados con el aumento de la cizalla (21).

el movimiento Browniano y la suspensión está líquida: el comportamiento es Newtoniano con baja viscosidad. Sin embargo, cuando se forman agregados por cambios en las condiciones del medio o bien porque el contenido en sólidos aumenta de forma que las partículas están tan próximas unas de otras que no se puede despreciar la influencia de las interacciones, la reología está gobernada por las fuerzas entre partículas y la suspensión se comporta virtualmente como un sólido presentando elasticidad y tensión de fluencia.

En la bibliografía se han propuesto numeras ecuaciones para relacionar la viscosidad aparente de las suspensiones con su fracción volumétrica de sólidos. Einstein (1956) fue el primero en obtener la relación entre la viscosidad relativa de la suspensión a bajas cizallas, $\eta_{\text {, }}$ y la fracción volumétrica de sólidos, $\phi$, para el sistema de esferas rígidas. En este sistema únicamente intervienen las fuerzas viscosas, el movimiento Browniano y el volumen de exclusión de las partículas, eliminando el problema de las interacciones entre partículas (22-25).

$$
\eta_{r}=\frac{\eta}{\eta_{L}}=1+[\eta] \cdot \phi
$$

siendo $\eta$ la viscosidad aparente de la suspensión, $\eta_{L}$ la viscosidad del fluido base y $\phi$ la fracción volumétrica de sólidos y $[\eta]$ se conoce como viscosidad intrínseca.

Batchelor (1977), considerando las contribuciones debidas a las interacciones hidrodinámicas y al movimiento Browniano, desarrolló una teoría para la viscosidad a bajas cizallas, para suspensiones diluidas de esferas rígidas:

$$
\eta_{r}=\frac{\eta}{\eta_{L}}=1+[\eta] \cdot \phi+K \cdot[\eta]^{2} \cdot \phi^{2}
$$

donde $K$ se conoce como coeficiente de Huggins.

Sin embargo, para altos contenidos en sólidos, el comportamiento de la viscosidad es difícil de predecir teóricamente debido a las múltiples interacciones. Para modelar el comportamiento de la viscosidad de suspensiones de esferas en la región de altas concentraciones, varias correlaciones de dos parámetros han sido propuestas.

La ecuación más utilizada ha sido la de Krieger-Dougherty (26), debido a que reproduce aceptablemente bien los 
resultados experimentales de numerosas suspensiones de partículas de características muy diferentes:

$$
\eta_{r}=\frac{\eta}{\eta_{L}}=\left(1-\frac{\phi}{\phi_{\max }}\right)^{-[\eta] \cdot \phi_{\max }}
$$

siendo $\phi_{\max }$ la fracción de empaquetamiento máxima.

Aunque la ecuación de Krieger-Dougherty se dedujo teóricamente para suspensiones de partículas esféricas del mismo tamaño y perfectamente estabilizadas, también se ha aplicado con éxito a suspensiones más complejas. Ahora bien, los valores de $[\eta]$ y $\phi_{\max }$ que para suspensiones de partículas gruesas, esféricas y monomodales valen $[\eta]=2,5 \mathrm{y}$ $\phi_{\max }=0,64$, para suspensiones de partículas no esféricas y/o polimodales, van a depender de algunas características de las partículas en suspensión, tales como la forma, orientación al flujo, amplitud de la distribución de tamaños y su estado de agregación. Se ha comprobado que la viscosidad intrínseca, [ $\eta$, depende considerablemente de la forma de las partículas o de los aglomerados, si estos últimos son las unidades de flujo. Su valor oscila entre 2,5 para partículas esféricas, hasta valores próximos a 14 para partículas aciculares altamente anisométricas.

Una expresión del mismo tipo que la de Krieger-Dougherty, pero con un valor fijo de $q=2$, fue obtenida por Quemada en 1977 (27) a partir de la aplicación del principio de mínima energía a la disipación de energía por efectos viscosos, y puede considerarse un caso particular de la relación general de Krieger-Dougherty.

$$
\eta_{r}=\frac{\eta}{\eta_{L}}=\left(1-\frac{\phi}{\phi_{\max }}\right)^{-2}
$$

Las suspensiones reales de nanopartículas estabilizadas electrostáticamente se pueden describir como sistemas de esferas rígidas sólo de forma aproximada, dependiendo del potencial de interacción. En el caso de un potencial repulsivo, es posible introducir un radio efectivo, $r_{\text {efp }}$ dependiendo sólo del rango de las interacciones electrostáticas, que define el radio equivalente de una esfera rígida como suma del radio de la partícula más el espesor de la doble capa eléctrica formada:

$$
r_{\text {eff }}=r_{p}+\mathrm{K}^{-1}
$$

Esta aproximación permite aplicar las ecuaciones anteriores sustituyendo el contenido en sólidos, $\phi$, por un contenido en sólidos efectivo, $\phi_{\text {eff }}(19,21,28)$.

$$
\phi_{\text {eff }}=\phi \cdot\left(\frac{r_{\text {eff }}}{r_{p}}\right)^{3}
$$

\subsection{Módulos elástico y viscoso}

Las suspensiones concentradas, cuando se someten a cizallas suficientemente pequeñas, pueden mostrar un comportamiento viscoelástico, que se caracteriza por la existencia de deformaciones viscosas y elásticas simultáneas. Cuando la contribución viscosa está representada por la ley de Newton y la elástica por la ley de Hooke, se dice que la suspensión presenta un comportamiento viscoelástico lineal (29).

La determinación del comportamiento viscoelástico de suspensiones suele realizarse mediante ensayos dinámicos oscilatorios. En este tipo de experimentos, la suspensión se somete a una tensión de cizalla, $\sigma$, cuya magnitud se modifica sinusoidalmente, y se registra la deformación producida, $\gamma$, también de tipo sinusoidal. Dicha función se descompone matemáticamente en otras dos funciones también sinusoidales de forma que, a partir de la primera se obtiene el módulo elástico o de almacenamiento, $G^{\prime}$, que describe la componente elástica del comportamiento del material. De la segunda, se obtiene el módulo viscoso o de pérdida, $G^{\prime \prime}$, que cuantifica la componente viscosa. Para un sólido elástico ideal $G^{\prime \prime}=0$ y $G^{\prime}$ está relacionada con la energía elástica almacenada en el material. Similarmente, para un fluido newtoniano $G^{\prime}=0$ y $G^{\prime \prime}$ está relacionado con la velocidad de disipación de energía, es decir, con la viscosidad. El comportamiento viscoelástico de las suspensiones reales es siempre intermedio entre dos situaciones ideales y extremas: el sólido viscoelástico de Kelvin y el líquido viscoelástico de Maxwell.

En el sólido viscoelástico de Kelvin todas las deformaciones son reversibles (elásticas), pero no son instantáneas. Para estos materiales, la variación con la frecuencia de los módulos elástico y viscoso es la siguiente:

$$
\begin{aligned}
& G^{\prime}(\omega)=G_{i} \\
& G^{\prime \prime}(\omega)=\eta_{i} \cdot \omega
\end{aligned}
$$

Según estas ecuaciones, para un sólido viscoelástico, $G^{\prime}$ es independiente de la frecuencia, mientras que $G^{\prime \prime}$ es proporcional. Por otra parte, las suspensiones floculadas y/o concentradas presentan este tipo de comportamiento y muestran valores de $G^{\prime}$ mucho mayores que los de G' (Figura 6a).

En el líquido viscoelástico de Maxwell la deformación total del sistema es la suma de una deformación elástica y una deformación viscosa la cual es permanente y dependiente del tiempo. Para estos materiales, para bajas frecuencias de oscilación, $G^{\prime}$ es proporcional a $\omega^{2}$ y $G^{\prime \prime}$ es proporcional a $\omega$ :

$$
\begin{aligned}
G^{\prime}(\omega) & =\frac{G_{i} \omega^{2} \lambda_{i}^{2}}{1+\omega^{2} \lambda_{i}^{2}} \\
G^{\prime \prime}(\omega) & =\frac{G_{i} \omega \lambda_{i}}{1+\omega^{2} \lambda_{i}^{2}}
\end{aligned}
$$

En general, las suspensiones bien desfloculadas tienen un comportamiento reológico que se aproxima al del líquido viscoelástico de Maxwell, y presenta valores de $G^{\prime \prime}$ mayores que los del G' (Figura 6b).

El comportamiento viscoelástico de las suspensiones coloidales depende de su estabilidad y del grado de agregación entre las partículas. El valor del módulo elástico indica la fuerza de las interacciones entre las partículas. Cuando las partículas están altamente aglomeradas la suspensión se comporta como un sólido: el módulo elástico es superior al viscoso y varían con la frecuencia de acuerdo con el sólido 


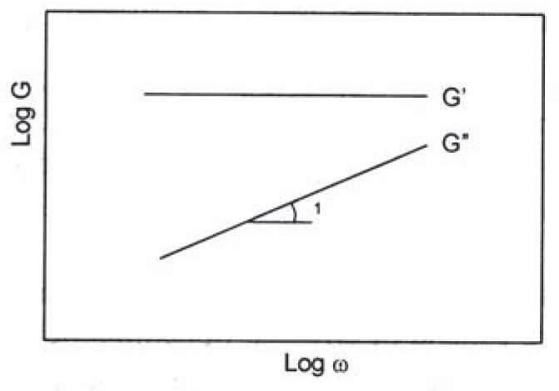

(a)

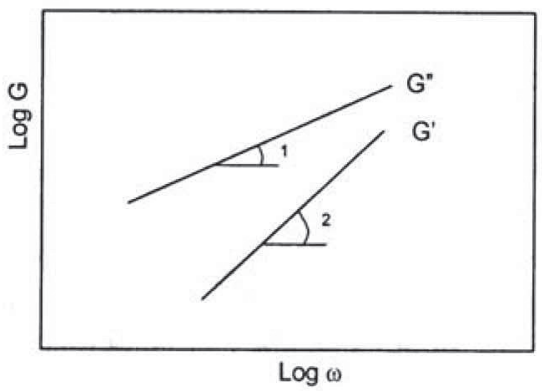

(b)

Figura 6. (a) Sólido viscoelástico de Kelvin. (b) Líquido viscoelástico de Maxwell.

viscoelástico de Kelvin. Por el contrario, suspensiones con un alto grado de dispersión presentan estructuras que fluyen comportándose como un líquido: el módulo elástico es inferior al viscoso y varían con la frecuencia de acuerdo con el líquido viscoelástico de Maxwell. En general, las suspensiones estables o con bajo contenido en sólidos se comportan como líquidos mientras que en aquellas condiciones que se produce la formación de agregados el comportamiento pasa de ser viscoso (como un líquido) a elástico (como un gel).

La influencia de la fracción volumétrica de sólidos sobre el comportamiento viscoelástico de las suspensiones coloidales depende de las interacciones entre las partículas. La respuesta viscoelástica de las suspensiones coloidales estabilizadas electrostáticamente tiene su origen en las interacciones repulsivas entre las partículas. Para concentraciones elevadas, próximas al máximo empaquetamiento, la superposición de los volúmenes de exclusión también determinará las propiedades elásticas del sistema.

En este tipo de sistemas, el módulo elástico, $G^{\prime}$, aumenta con la fracción volumétrica de sólidos. Al aumentar la fracción volumétrica de sólidos, disminuye la distancia media de separación entre las partículas, lo que provoca una superposición cada vez mayor de las dobles capas. Este comportamiento se refleja en un aumento considerable del módulo elástico para valores de $\phi$ elevados. Para valores bajos, no se produce la superposición de las dobles capas de forma apreciable, por lo que el sistema presenta un comportamiento exclusivamente viscoso. La variación de los módulos $G^{\prime}$ y $G^{\prime \prime}$ con el contenido en sólidos se puede modelizar utilizando relaciones de tipo potencial, del mismo tipo que la de Krieger-Dougherty.

\subsection{Potencial zeta}

El potencial zeta es el potencial de las partículas en el plano de cizalla, es decir, en la superficie exterior de la partícula considerando la capa de contraiones que se desplaza junto a ella en caso de existir (6).

Las medidas de potencial zeta proporcionan un análisis inmediato del grado de estabilidad y dispersión de las partículas en suspensión. Se trata de una medida directamente proporcional a la carga de las partículas, de forma que cuanto mayor sea su valor, más cargadas estarán éstas y mayor será el potencial electrostático y la consecuente repulsión que impedirá que las partículas se atraigan y permanezcan unidas formándose agregados.
El potencial zeta toma el valor cero en el punto isoeléctrico, el cual no existen fuerzas electrostáticas, mientras que aumenta (en el sentido positivo o negativo) cuando el $\mathrm{pH}$ se aumenta o disminuye con respecto a dicho punto. Por otra parte, la adición de un electrolito disminuye el valor del potencial zeta ya que los contraiones son adsorbidos sobre la superficie de la partícula provocando un efecto de apantallamiento que disminuye el valor de la carga neta (9). En general, se considera que una suspensión es inestable para valores de potencial zeta inferiores a $25 \mathrm{mV}$, mientras que para valores entre 40 $\mathrm{mV}$ y $60 \mathrm{mV}$ ya se puede considerar que la estabilidad de la suspensión es buena. Las mejores condiciones de estabilidad son aquellas en que el potencial zeta es superior a $60 \mathrm{mV}$.

\subsection{Tamaño de partícula}

El tamaño de partícula o agregado puede utilizarse como indicador macroscópico de las interacciones entre partículas. De esta forma, el tamaño de agregado disminuye a medida que la barrera energética aumenta y se dificulta el proceso de agregación de las partículas. Por tanto el tamaño de clúster formado depende también de las condiciones de preparación de la suspensión y se ve influenciado por muchos factores como el contenido en sólidos, el $\mathrm{pH}$, la fuerza iónica, la energía de dispersión y el tiempo.

El proceso de desaglomeración tiene lugar en dos pasos. En el primer paso, los agregados grandes del polvo inicial (entre 5 y $500 \mu \mathrm{m}$ ) se rompen en agregados secundarios más pequeños de tamaño entre 2 y $80 \mu \mathrm{m}$. La distribución de tamaños, que en principio es monomodal, se desplaza hacia tamaños cada vez menores a medida que avanza el proceso. La forma de la distribución indica que en este paso la fragmentación de agregados secundarios es el principal mecanismo de desagregación. En el segundo paso, los agregados secundarios grandes empiezan a romperse en agregados primarios de menor tamaño. En este punto, la distribución pasa a ser bimodal, existiendo un primer modo representativo de los agregados primarios, y un segundo modo representativo de los agregados secundarios. Un aumento del tiempo o la energía de desagregación produce una reducción gradual de la fracción de agregados en el segundo modo y un aumento de la fracción del primer modo. En este momento la distribución pasa a ser monomodal de nuevo, existiendo en este caso agregados primarios de menor tamaño $(120 \mathrm{~nm})$. Estos agregados primarios no pueden 
TABLA I. TRABAJOS PREVIOS DE DISPERSIÓN Y CARACTERIZACIÓN.

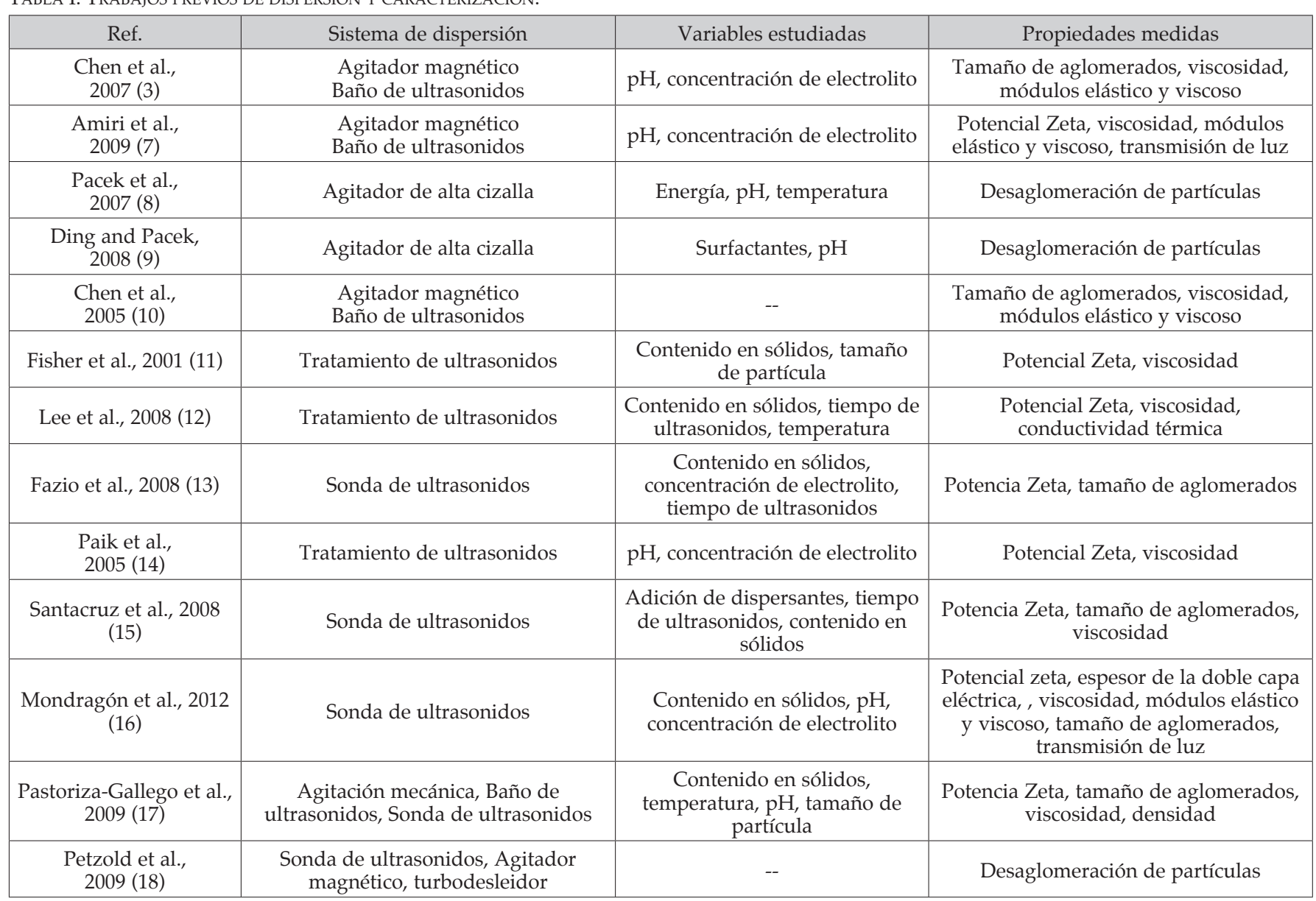

romperse en las partículas primarias incluso a la energía más alta posible $(7,18,30)$.

Los agregados pueden romperse por tensión normal o cizalla por erosión, o por ruptura de toda la masa. El mecanismo de ruptura depende del tamaño de los agregados y de la intensidad de energía aplicada. A medida que los agregados se vuelven más pequeños conforme avanza el proceso de desaglomeración, las fuerzas superficiales adquieren mayor importancia. Por lo tanto, la ruptura de los agregados grandes es relativamente simple, mientras que la ruptura de agregados menores de $1 \mu \mathrm{m}$ puede resultar muy complicada, llegando las partículas en 10 y $100 \mathrm{~nm}$ a no poder ser separadas por acción mecánica.

\subsection{Transmisión de luz}

Los tests de transmisión de luz se usan como medida de la agregación por la interacción entre las partículas. Durante la coagulación, por definición, las partículas se concentran en agregados con un índice de refracción mayor que el del medio $(6,31)$. Por tanto, la coagulación va seguida de un aumento en la turbidez y una disminución en la transmisión de luz. Cuanto mayor sea el nivel de agregación, mayor es la turbidez de la muestra y menor la transmisión de luz de la misma.

Por otra parte, a partir de la evolución de la transmisión de luz con el tiempo se puede analizar el proceso de floculación y determinar la estabilidad del sistema. Un valor constante de la transmisión de luz durante todo el periodo de tiempo revela el dominio de la estabilidad de la suspensión. Por el contrario, conforme se produce la formación de agregados la transmisión de luz disminuye progresivamente. Al realizar la medida en un punto de la muestra situado en la parte superior, si la transmisión disminuye alcanzando un valor asintótico, se puede decir que la suspensión se comporta como un gel con estructura tridimensional. En cambio, si la disminución inicial de transmisión, debido a la aglomeración de las partículas, va seguida de un aumento de transmisión, debido a la clarificación del líquido, se puede concluir que se ha producido una separación de fases provocada por la floculación y posterior precipitación de los flóculos.

En la Tabla I se resumen los principales trabajos sobre preparación y caracterización de nanofluidos. En ella se muestra el sistema de dispersión utilizado para dispersar las partículas en el medio, las variables analizadas y las propiedades medidas para llevar a cabo la caracterización de las suspensiones y estudiar cómo se modifican estás al variar las condiciones de preparación.

\section{CONCLUSIONES}

El uso de nanofluidos resulta de gran interés en numerosas aplicaciones debido a las ventajas que presenta con respecto de las suspensiones conteniendo materiales de tamaño 
micrométrico: elevada superficie específica, reducido tamaño que impide la obturación de conductos, y sobre todo, una elevada estabilidad. El hecho de que las suspensiones puedan permanecer durante periodos prolongados de tiempo sin sedimentar es una de los principales beneficios derivados del uso de nanofluidos.

Para poder obtener suspensiones altamente estables, es necesario conocer en detalle cuales son las variables de la suspensión que influyen en el estado de aglomeración de las partículas, y por lo tanto, en la estabilidad. Estás variables son el $\mathrm{pH}$ del medio, la presencia de electrolitos y el contenido en sólidos. Así pues, un valor de $\mathrm{pH}$ alejado del punto isoeléctrico del material asegura una elevada repulsión electrostática entre las partículas y mayor estabilidad. Por el contrario, la presencia de electrolitos disminuye el espesor de la doble capa eléctrica retrasando el momento en que se produce la repulsión, favoreciendo la aglomeración. Todas las interacciones se ven potenciadas al aumentar el contenido en sólidos de la suspensión y disminuir la distancia entre las partículas.

Otro de los factores importantes es el método utilizado para dispersar las nanopartículas en el medio líquido. Existen diversos sistemas de dispersión, los cuales proporcionan niveles de energía diferentes. Para conseguir el mayor grado de dispersión posible es necesario hacer uso de sistemas que aporten una elevada energía y permitan disgregar los aglomerados de nanopartículas lo máximo posible, en el menor tiempo. En este sentido, las sondas de ultrasonidos son las más eficientes.

Finalmente, el estado de aglomeración y la estabilidad de los nanofluidos puede determinarse a partir de las propiedades que se ven modificadas cuando varían las condiciones de la suspensión. Estas propiedades son la viscosidad y el comportamiento reológico (Newtoniano o pseudoplástico), el comportamiento viscoelástico (como sólido o como líquido), el potencial zeta, el tamaño de los aglomerados y la variación de la cantidad de luz retrodispersada o transmitida por la muestra con el tiempo.

\section{BIBLIOGRAFÍA}

1. H.E. Bergna, W.O. Roberts. Colloidal silica: Fundamentals and applications. CRC Taylor \& Francis. 2006.

2. U.S. Choi. Enhancing thermal conductivity of fluids with nanoparticles. ASME FED 231, 99-103, 1995.

3. D. Quemada, C. Berli. Energy of interaction in colloids and its implication in rheological modelling. Adv Colloid Interf Sci 98, 51-85, 2002.

4. R.J. Hunter. Zeta potential in colloid science. Principles and applications. Academic Press Limited. 1988.

5. S. Song, C. Peng. Thickness of solvation layers on nano-scale silica dispersed in water and ethanol. J Disp Sci Technol 26, 197-201, 2005.

6. A. Amiri, G. Oye, J. Sjöblom. Influence of $\mathrm{pH}$, high salinity and particle concentration on stability and rheological properties of aqueous suspensions of fumed silica. Colloids Surf A: Physicochem Eng Aspects 349, 43-54, 2009.
7. A.W. Pacek, P. Ding, A.T. Utomo. Effect of energy density, $\mathrm{pH}$ and temperature on de-aggregation in nano-particles/water suspensions in high shear mixer. Powder Technol 173, 203-210, 2007.

8. P. Ding, A.W. Pacek. De-agglomeration of silica nanoparticles in the presence of surfactants. J Disp Sci Technol 29, 593-599, 2008.

9. S. Chen, G. Oye, J. Sjöblom. Effect of $\mathrm{pH}$ and salt on rheological properties of aerosol suspensions. J Disp Sci Technol 28, 845-853, 2007.

10. S. Chen, G. Oye, J. Sjöblom. Rheological properties of aqueous silica particle suspensions. J Disp Sci Technol 26, 495-501, 2005.

11. M.L. Fisher, M. Colic, M.P. Rao, F.F. Lange. Effect of silica nanoparticles size on the stability of alumina/silica suspensions. J Am Ceram Soc 84, 713-718, 2001.

12. J-H. Lee, K.S. Hwang, S.P. Jang, B.H. Lee, J.H. Kim, S.U.S. Choi, C.J. Choi. Effective viscosities and thermal conductivities of aqueous nanofluids containing low volume concentrations of $\mathrm{Al}_{2} \mathrm{O}_{3}$ nanoparticles. Int J Heat Mass Transf 51, 2651-2656, 2008.

13. S. Fazio, J. Guzman, M.T. Colomer, A. Salomoni, R. Moreno. Colloidal stability of nanosized titania aqueous suspensions. J Eur Ceram Soc 28, 2171-2176, 2008.

14. U. Paik, J. Yul Kim, V.A. Hackley. Rheological and electrokinetic behaviour associated with concentrated nanosized silica hydrosols. Mater Chem Phys 91, 205-211, 2005.

15. I. Santacruz, K. Anapoorani, J. Binner. Preparation of high solid content nanozirconia suspensions. J Am Ceram Soc 91, 398-405, 2008.

16. R. Mondragón, J.E. Juliá, A. Barba, J.C. Jarque. Characterization of silicawater nanofluids dispersed with an ultrasound probe: a study of their physical properties and stability. Powder Technol 224, 138-146, 2012.

17. M.J. Pastoriza-Gallego, C. Casanova, R. Paramo, B. Barbes, J.L. Legido M.M. Piñeiro. A study on stability and thermophysical properties (density and viscosity) of $\mathrm{Al}_{2} \mathrm{O}_{3}$ in water nanofluid. J Appl Phys 106, 064301, 2009

18. G. Petzold, R. Rojas-Reyna, M. Mende, S. Schwarz. Application relevant characterization of aqueous silica nanodispersions. J Disp Sci Technol 30, 1216-1222, 2009.

19. J.L. Amoros, V. Berltran, V. Sanz, J.C. Jarque. Electrokinetic and rheological properties of highly concentrated kaolin dispersions: influence of particle volume fraction and dispersant concentration. Appl Clay Sci 49, 33-43, 2010.

20. H.A. Barnes, J.F. Hutton, D.K. Walters. An introduction to rheology. Elsevier. 1989.

21. D. Quemada. Rheological modelling of complex fluids. I. The concept of effective volume fraction revised. Eur Phys J - Appl Phys 1, 119-127, 1998.

22. I.R. Rutgers. Relative viscosity and concentration. Rheol Acta 2, 305-348, 1962a.

23. I.R. Rutgers. Relative viscosity of suspensions of rigid spheres in Newtonian liquids. Rheol Acta 2, 202-210, $1962 \mathrm{~b}$.

24. F.J. Rubio-Hernandez, M.F. Ayucar-Rubio, J.F. Velazquez-Navarro, F.J Galindo-Rosales. Intrinsic viscosity of $\mathrm{SiO}_{2}, \mathrm{Al}_{2} \mathrm{O}_{3}$ and $\mathrm{TiO}_{2}$ aqueous suspensions. J Colloid Interf Sci 298, 967-972, 2006.

25. C.J. Rueb. Flow properties of a colloidal gel. Universidad de Illinois. Tesis Doctoral. 1994.

26. I.M. Krieger, T.J. Dougherty. A mechanism for non-Newtonian flow in suspensions of rigid spheres. Trans Soc Rheol 3, 137-152, 1959.

27. D. Quemada. Rheology of concentrated disperse systems and minimum energy dissipation principle. I. Viscosity-concentration relationship. Rheol Acta 16, 82-94, 1977.

28. R. Lapasin, M. Grassi, S. Pricl. Rheological modelling of fractal and dense suspensions. Chem Eng J 64, 99-106, 1996.

29. V. Sanz. Comportamiento reológico de las suspensiones arcillosas concentradas. Universitat Jaume I. Tesis Doctoral. 2001.

30. P. Ding, M.G. Orwa, A.W. Pacek. De-agglomeration of hydrophobic and hydrophilic silica nano-powders in a high shear mixer. Powder Technol 195, 221-226, 2009.

31. R.K. Iler, The chemistry of silica: solubility, polymerization, colloid and surface properties, and biochemistry. John Wiley \& Sons. 1979.

Recibido:03/09/2013

Recibida versión corregida: $02 / 12 / 2013$

Aceptado: 04/12/2013 